\title{
Status of $\mathbf{R}$ value measurement at BESIII
}

\author{
Haiming $\mathrm{HU}^{1, *} \quad$ (for the BESIII Collaboration) \\ ${ }^{1}$ Institute of High Energy Physics, CAS, Beijing
}

\begin{abstract}
The dominant systematic error of $\mathrm{R}$ value measurement comes from the hadronic generator simulations. This paper reviews the present status of $\mathrm{R}$ value measurement from 2.232 to $3.671 \mathrm{GeV}$ at 14 energy points, especially describes the calculations of initial state radiative correction and the issue of the parameter tuning for the Lund area law generator LUARLW.
\end{abstract}

\section{Introduction}

$\mathrm{R}$ value is defined as the inclusive $e^{+} e^{-}$annihilation hadronic production cross section at the tree level Feynman diagram normalized by the theoretical di-muon cross section. $\mathrm{R}$ value is a basic input parameter for the calculations of the abnormal magnetic moment of muon $(g-2)_{\mu}$, and the QED running coupling constant $\alpha(s)$. In fact, the hadronic contribution for the calculations of $(g-2)_{\mu}$ and $\alpha(s)$ are the infinite integrals about energy from $2 m_{\pi}$ to $\infty$ $\mathrm{GeV}$, the hadronic cross section ( $\mathrm{R}$ value) below $5 \mathrm{GeV}$ adopts the experimental value, and in higher energy region the hadronic cross section use the value predicted by $\mathrm{pQCD}$. So, the experimental errors of $\mathrm{R}$ value, especially the $\mathrm{R}$ value errors below $3 \mathrm{GeV}$, will result in the dominant uncertainties of $(g-2)_{\mu}$ and $\alpha(s)[1-3]$.

BESII ever measured $\mathrm{R}$ value between $2-5 \mathrm{GeV}$. In references $[4,5]$, the $\mathrm{R}$ values between 2-5 GeV were measured with average error of about 7\%, and in reference [6] the errors of $\mathrm{R}$ value were about $3.5 \%$. Recently, KEDR published the $\mathrm{R}$ value below open charm with improved accuracy for the measurement with small statistical data[7]. To reduce the error of $\mathrm{R}$ value is still one of the experimental goals at BESIII.

The data samples at 130 energy points from 2.0 to $4.59 \mathrm{GeV}$ have been collected with BESIII, the total integrated luminosity is about $1.3 \mathrm{fb}^{-1}$. This note will focuses on the issue of the initial state radiative corrections and the simulation of hadronic events by the Lund area law generator LUARLW and parameters tuning with BESIII data.

\section{Data analysis}

In experiment, $R$ value is measured with following expression:

$$
R_{\text {exp }}=\frac{N_{h a d}^{o b s}-N_{b g}}{\sigma_{\mu \mu}^{0} L \epsilon_{t r g} \bar{\epsilon}_{h a d}(1+\delta)},
$$

the meanings of all of these quantities in above formula were explained in references [4-6].

\footnotetext{
*e-mail: huhm@ihep.ac.cn
} 
The basic physics processes produced in $e^{+} e^{-}$annihilation in BEPCII energy region are four types: $e^{+} e^{-} \rightarrow l^{+} l^{-}, \gamma \gamma, e^{+} e^{-} X$ and hadrons. The scheme of event selection in this work are similar to the criteria described in references [4-6]. The numbers of the residual QED background events $N_{b g}$ in Eq.(1) are statistically subtracted by MC method:

$$
N_{b g}=L\left[\epsilon_{e e} \sigma_{e e}+\epsilon_{\mu \mu} \sigma_{\mu \mu}+\epsilon_{\tau \tau} \sigma_{\tau \tau}+\epsilon_{\gamma \gamma} \sigma_{\gamma \gamma}\right]
$$

where $L$ is the integrated luminosity of data, $\sigma_{e e}$ the cross section of Bhabha process, $\epsilon_{e e}$ the residual efficiency of Bhabha process which passes the hadronic event selection criteria, other symbols have corresponding meanings.

\section{Initial state radiation}

The directly measured hadronic cross section is the observed total cross section, which contains the total contributions of all Feynman diagrams shown in Figure 1:
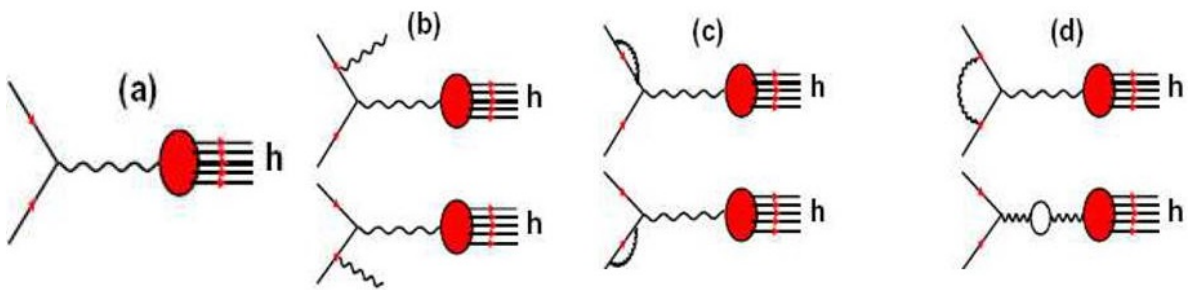

Figure 1. The Feynman diagrams of $e^{+} e^{-}$annihilation into hadrons up to one-loop order: (a) the tree level diagram, (b) the bremsstrahlung, (c) electron self-energy, (d) vertex correction and vacuum polarization.

The $R$ value is the Born cross section corresponding to the tree level Feynman diagram of Figure 1(a). The fraction contribution of the higher level diagrams in Figure 1(b), (c) and (d) can be calculated by the initial state radiative correction:

$$
\sigma^{t o t}(s)=(1+\delta) \sigma^{0}(s), \text { or }(1+\delta)=\frac{\sigma^{t o t}(s)}{\sigma^{0}(s)},
$$

where $\sigma^{0}(s)$ is the Born cross section, $\sigma^{\text {tot }}(s)$ the total cross section, and $(1+\delta)$ is called the ISR correction factor, which reflects the fraction of the contributions of the higher level processes.

Total cross section $\sigma^{\text {tot }}(s)$ can be calculated by the initial state radiation correction theory at one-loop approximation of order $O\left(\alpha^{3}\right)$ introduced in references [11-13], but expressed in an improved form:

$$
\sigma^{t o t}(s)=\left(1-x_{m}^{\beta}+\delta_{v e r t}\right) \frac{\sigma^{0}(s)}{|1-\hat{\Pi}(s)|^{2}}+\int_{0}^{x_{m}} d x x^{\beta} F_{F D}(x) \frac{\sigma_{0}\left(s^{\prime}\right)}{\left|1-\hat{\Pi}\left(s^{\prime}\right)\right|^{2}},
$$

where $x=2 E_{\gamma} / E_{c m}$ is the energy fraction carried away by the bremsstrahlung photon, $x_{m}=$ $1-4 m_{\pi}^{2}$ the maximum value of $x, s^{\prime}=(1-x) s$ the squared effective energy of the hadronic final state, $\delta_{\text {vert }}$ the initial vertex correction factor, $\hat{\Pi}(s)$ the vacuum polarization correction in the 1-particle-irreducible (1PI) chain approximation, and $F_{F D}$ the radiator. The Figure 2 (left) shows the numerical ISR factor $(1+\delta)$ calculated by the Feynman diagram scheme Eq.(4) and the structure function scheme[14, 15]. The numerical results of $(1+\delta)$ calculated by Feynman diagram and structure function schemes agree within $1 \%$. 

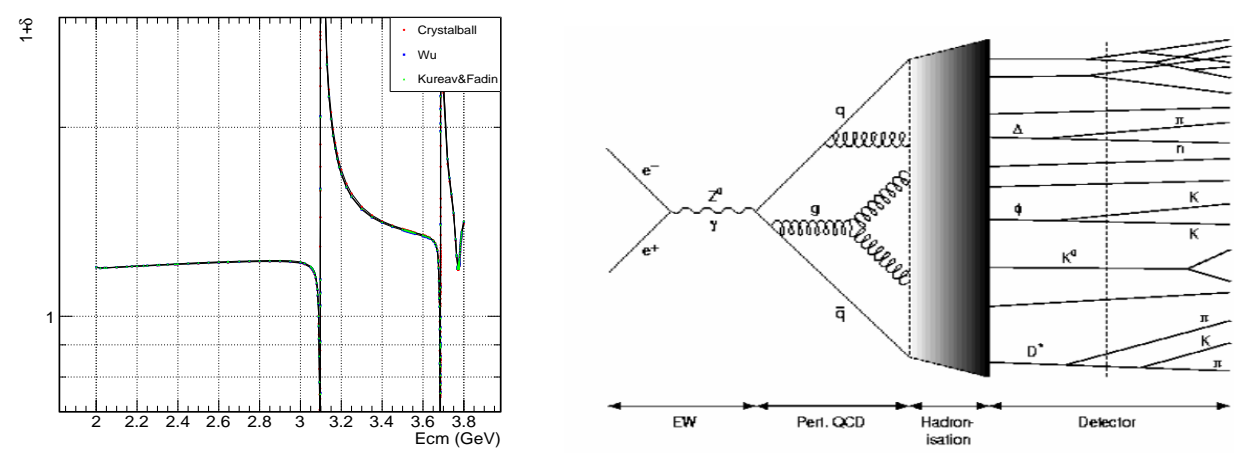

Figure 2. Left: the ISR correction factor $(1+\delta)$ calculated by Feynman diagram scheme (Crystalball) and structure function schemes (KureavFadin, Wu). Right: the picture of $e^{+} e^{-}$annihilation into hadrons.

\section{Generator LUARLW}

Figure 2 (right) shows the general picture of $e^{+} e^{-}$annihilation and hadrons production. The nonperturbative hadronization process can be described by the Lund string fragmentation model[8]. The Lund area law generator LUARLW[9, 10] is built in order to simulate the few-body production processes in BEPC energy region. The maximum number of primary hadrons in string fragmentation is six. LUARLW simulation contains following constitutes: initial state radiation (ISR), string fragmentation, multiplicity and momentum-energy distributions, decay of unstable hadrons.

\subsection{Simulation of ISR return processes}

In the simulations of ISR return processes, the hadronic process are divided into two classes: the virtual radiative corrections and real bremsstrahlung, and their weights are:

$$
\begin{aligned}
\sigma^{V S B}(s) & =\left[1+\beta \ln \left(x_{0}\right)+\delta_{A R}\right] \sigma^{0}(s), \\
\sigma^{H B}(s) & =\int_{0}^{4 \pi} d \Omega_{\gamma} \int_{x_{0}}^{x_{m}} d x \frac{d \sigma^{H B}(x, \theta)}{d x d \Omega_{\gamma}}
\end{aligned}
$$

The meanings of $x_{0}, \beta$ and $\delta_{A R}$ were explained in [11-13]. A bremsstrahlung event which with the radiative photon angles $(\theta, \phi)$ and fraction momentum $x$ can be sampled by the differential cross section:

$$
\frac{d \sigma^{H B}(x, \theta)}{d x d \Omega_{\gamma}}=\frac{\alpha}{\pi^{2}} \frac{\sin ^{2} \theta}{\left(1-a^{2} \cos ^{2} \theta\right)^{2}} \frac{1}{x}\left(1-x+\frac{x^{2}}{2}\right) \sigma^{0}\left(s^{\prime}\right)
$$

The values of $\sigma^{0}(s)$ from $2 m_{\pi}$ to $1.8 \mathrm{GeV}$ use experimental values cited in PDG [16], and in the energy region above $1.8 \mathrm{GeV}$,

$$
\sigma^{0}(s)=\sigma_{\mu}^{0}(s) R_{p Q C D}(s)+\sigma_{B W}(s),
$$

where $\sigma_{\mu}^{0}(s)$ is the theoretical di-muon Born cross section, $R_{p Q C D}(s)$ the R value of continuous hadronic states predicted by pQCD, $\sigma_{B W}(s)$ the resonant cross section calculated by the BreitWigner formula. The Figure 3 shows the number of events distribution with different effective hadronic energies $\sqrt{s^{\prime}}$ in LUARLW sampling. The peaks of resonances $\rho, \phi$ and $J / \psi$ which with larger Breit-Wigner cross sections are clearly visible. Correctly simulating the ISR return processes are important for reducing the systematic error of efficiency $\bar{\epsilon}_{h a d}$. 

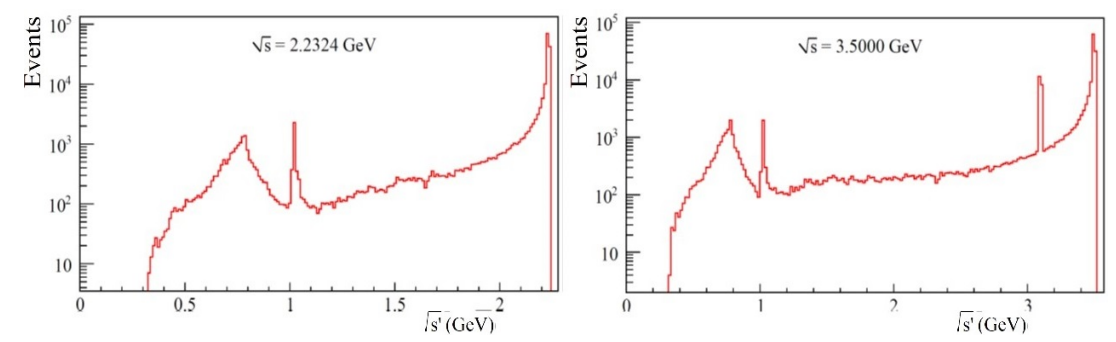

Figure 3. The event distributions of ISR return processes with different effective hadronic energies $\sqrt{s^{\prime}}$.

\subsection{Simulation of hadronic processes}

\subsubsection{Physics picture}

The basic physics picture of LUARLW is the Lund string fragmentation model[8, 9]. Figure 5 shows that mesons $(M)$ and baryons ( $B$ and $\bar{B}$ ) produce at the vertex of light-cone area in string fragmentation processes. Single-quark-pair excitation in string fragmentation form mesons, and di-quark-pair excitation form baryons.

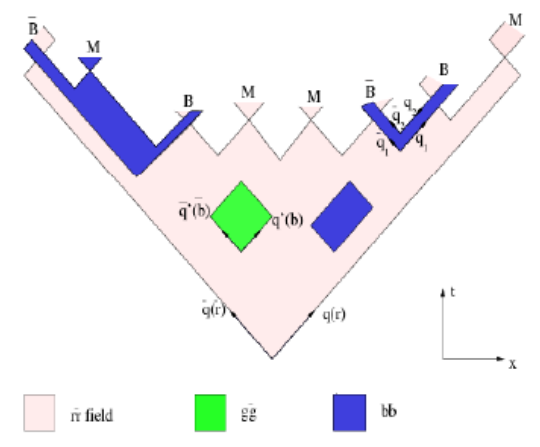

Figure 4. The mesons (M) and baryons (B) production in the string fragmentation processes.

Starting from the Lund area law, one may obtain an approximation expression of poissonlike multiplicity distribution of the preliminary fragmentation hadrons[10]:

$$
P_{n}(s)=\frac{\mu^{n}}{n !} \exp \left[c_{0}+c_{1}(n-\mu)+c_{2}(n-\mu)^{2}\right]
$$

where $n$ is the number of the hadrons, and the parameter $\mu$ can be understood as the average multiplicity. The energy dependence of $\mu$ can approximately quote the QCD-like prediction:

$$
\mu=\alpha+\beta \exp (\gamma \sqrt{s})
$$

where $c_{1}, c_{2}, c_{3}, \alpha, \beta$ and $\gamma$ are free parameters.

\subsubsection{Production modes}

The simulations of the continuum states include the lowest and leading order QCD correction:

$$
e^{+} e^{-} \rightarrow \gamma^{*} \rightarrow\left\{\begin{array}{l}
q \bar{q} \rightarrow \text { string } \rightarrow \text { hadrons } \\
g q \bar{q} \rightarrow 2 \text { strings } \rightarrow \text { hadrons }
\end{array}\right.
$$


For the 2-string mode, each string fragments independently according to the Lund area law. The probability of an exclusive process $e^{+} e^{-} \rightarrow q \bar{q}(g) \rightarrow \operatorname{string}(\mathrm{s}) \rightarrow m_{1}+m_{2} \ldots+m_{n}$ can be factorized as:

$$
d \sigma_{n}(s)=d \sigma\left(e^{+} e^{-} \rightarrow q \bar{q}\right) \cdot d \mathcal{P}_{n}\left(q \bar{q}(g) \rightarrow m_{1}, m_{2} \ldots m_{n} ; s\right),
$$

where $d \sigma\left(e^{+} e^{-} \rightarrow q \bar{q}\right)$ is the QED cross section, $d \mathcal{P}_{n}$ the probability for string fragmentation into $n$ hadrons. The energy-momentum distributions are determined by Lund area law[10].

The vector mesons whose masses smaller than $2 \mathrm{GeV}$ and with quantum number $J^{P C}=$ $1^{--}$can directly couple to virtual photon in ISR return process:

$$
e^{+} e^{-} \rightarrow \gamma^{*} \rightarrow \rho(770), \omega(782), \phi(1020) \cdots \rho(1700) .
$$

The decay fractions of these vector mesons to different final states take PDG values, and the polar angles distribution depends on their decay final states[17].

The production and decay of the charmonium adopt the standard pictures[18, 19]. For example, the simulation of $J / \psi$ contain following channels:

$$
e^{+} e^{-} \rightarrow \gamma^{*} \rightarrow J / \psi \rightarrow\left\{\begin{array}{l}
\gamma^{*} \rightarrow e^{+} e^{-}, \mu^{+} \mu^{-} \\
\gamma^{*} \rightarrow q \bar{q} \rightarrow \text { string } \rightarrow \text { hadrons } \\
g g g \rightarrow 3 \text { strings } \rightarrow \text { hadrons } \\
\gamma g g \rightarrow 2 \text { strings } \rightarrow \text { hadrons } \\
\gamma \eta_{c} \rightarrow g g \rightarrow 2 \text { strings } \rightarrow \text { hadrons } \\
\gamma+\text { radiative decay channels (cite PDG values) }
\end{array}\right.
$$

The simulations for $\psi(3686), \psi(3770), \psi(4040), \psi(4190)$ and $\psi(4415)$ are in the similar ways.

\subsection{Parameter tuning of LUARLW}

Two schemes are used to tune the parameters of LUARLW, which are described below.

\subsubsection{Scheme A: hybrid PHOKHARA+ConExc+LUARLW}

The red points in Figure 5 (left) show the sum of cross sections of the measured exclusive processes, and the black points are the total cross sections measured inclusively.

PHOKHARA+ConExc+LUARLW is a hybrid generator. The processes ever measured exclusively are simulated by the exclusive package PHOKHARA[21-23] or ConExc[24], and the remaining unmeasured processes are simulated by LUARLW in inclusive way. There is no free parameter in PHOKHARA and ConExc, all free parameters are in LUARLW.

\subsubsection{Scheme B: Pure LUARLW}

In this scheme, LUARLW is used an independent generator. The main parameters to be tuned are those about the multiplicity of the preliminary hadrons in Eq.(9) and Eq.(10), and those which determine the ratios of mesons and baryons with different quantum number $(S, L, J)$. In LUARLW these parameters are stored in array PARJ(1-20) as did in JETSET[20]. The parameters tuning is in progress.

\section{Summary}

The data analysis of $\mathrm{R}$ value measurement almost has been finished. The challenging for the $\mathrm{R}$ value measurement is still parameters tuning of LUARLW, which is in progress. The goal is MC simulations agreeing BESIII data well, and the error of hadronic efficiency reach a acceptable level, for example $2 \%$. The total errors of $\mathrm{R}$ value reduce to about $3 \%$. 

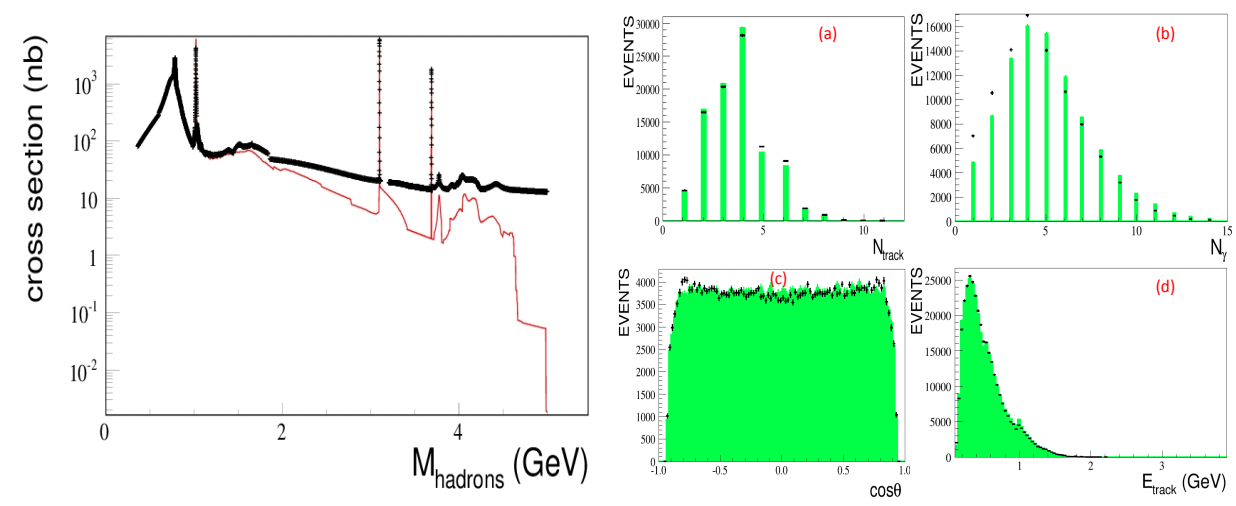

Figure 5. Left: the Born cross section of $e^{+} e^{-} \rightarrow$ hadrons below $5 \mathrm{GeV}$. The black points are the total cross section measured in inclusive method, the red points are the sum of the measured exclusive cross sections. Right: Comparison between data and MC in scheme A at $3.65 \mathrm{GeV}$. (a) multiplicity of charged track; (b) multiplicity of photon; (c) polar angle $\cos \theta$; (d) momentum of charged track.

\section{References}

[1] B.Pietrzyk, Nucl. Phys. B162 (Proc. Suppl.), 18 (2006)

[2] F. Jegerlehner, Nucl. Phys. B162 (Proc. Suppl.), 22 (2006)

[3] F. Jegerlehner, hep-ph/0104304

[4] BES Collaboration, J.Z.Bai et al., Phys. Rev. Lett. 84, 594 (2000)

[5] BES Collaboration, J.Z.Bai et al., Phys. Rev. Lett. 88, 101802 (2002)

[6] BES Collaboration, M. Ablikim et al., Phys. Lett. B677, 239, (2009)

[7] V.V. Anashin, et al., Phys. Lett. B 788, 42 (2019)

[8] Bo Andersson, The Lund Model (Cambrindge University Press, 1998)

[9] B. Andersson and H. Hu, hep-ph/9810285

[10] H. Hu et al., High Energy Physics and Nuclear Physics, 25, 1035 (2001) (in Chinese)

[11] A. Osterheld el al., SLAC-PUB-4160 (1986)(T/E)

[12] C. Edward el al., SLAC-PUB-5160 (1990)(T/E)

[13] H. Hu et al., High Energy Physics and Nuclear Physics, 25, 701 (2001) (in Chinese)

[14] E.A. Kureav and V.S. Fadin, Sov. J. Nucl. Phys. 41, 466 (1985)

[15] J. Wu, Chinese Physics C (HEP NP) 14, 585 (1990) (in Chinese)

[16] Paticle Data Group, Chinese Physics C, 38, 2014

[17] N. Cabibbo and R. Gatto, Phys. Rev. 124, 1577 (1961)

[18] L. Köpke and N. Wermes, Phys. Reprt. 17467 (1989)

[19] H. Hu et al., High Energy Physics and Nuclear Physics, 27, 673 (2003) (in Chinese)

[20] T.Sjöstrand, CERN-TH.7112/93, Decenber 1993

[21] G.Rodrigo et al., Eur. Phys. J. C24, 71 (2002)

[22] H.Czyz et al., Eur. Phys. J. C27, 563 (2003)

[23] H.Czyz et al., hep-ph/0308312

[24] R.Ping, Chin. Phys. C40, 113002 (2016) 